\title{
Strictly Finite-Time-Convergent Missile Guidance Law Based on Adaptive-Gain Observer
}

\author{
Jun Zhou and Yang Wang \\ Institute of Precision Guidance and Control, Northwestern Polytechnical University, Xian 710072, China \\ Correspondence should be addressed to Yang Wang; 2013100280@mail.nwpu.edu.cn
}

Received 8 November 2016; Revised 2 January 2017; Accepted 10 January 2017; Published 1 February 2017

Academic Editor: M. Djemai

Copyright (C) 2017 Jun Zhou and Yang Wang. This is an open access article distributed under the Creative Commons Attribution License, which permits unrestricted use, distribution, and reproduction in any medium, provided the original work is properly cited.

\begin{abstract}
In the absence of the upper bound of time-varying target acceleration, the finite-time-convergent guidance (FTCG) problem for missile is addressed in this paper. Firstly, a novel adaptive finite-time disturbance observer (AFDO) is developed based on adaptivegain super twisting (ASTW) algorithm to estimate the unknown target acceleration. Subsequently, a new FTCG law is proposed by using the output of AFDO. The newly proposed FTCG law has several advantages over existing FTCG laws. First, for timevarying target acceleration, the proposed method can strictly guarantee the trajectory of the closed-loop system is driven onto the sliding surface rather than a neighbourhood of sliding surface in the extended-state-observer-based FTCG (ESOFTCG) law. Second, the proposed method requires no upper bound information on the target acceleration. Third, the chattering problem in the conventional FTCG (CFTCG) law is completely avoided in this paper. Simulation result demonstrates the effectiveness of the proposed AFDO and the proposed FTCG law.
\end{abstract}

\section{Introduction}

As a classical guidance method, proportional navigation guidance law (PNGL) [1-6] has the advantage of easy implementation in engineering. However, a series of theoretical researches and engineering practices have shown that PNGL has insufficient effect in the presence of maneuvering target. To improve the robustness of guidance system in allusion to maneuvering target, many modern control theories have been applied to design guidance laws, such as nonlinear $H_{\infty}$ robust guidance law [7], L2 gain guidance law [8], Lyapunovtheory-based nonlinear guidance law [9], and sliding-mode guidance law [10]. However, the missile guidance problem considered in [7-10] is solved by the asymptotic stability analysis which implies that the system trajectories converge to the equilibrium with infinite time. Actually, in many applications, the time of termination is really quite short. For example, in the space interception where a missile is intercepting a ballistic target, the whole process of terminal guidance usually lasts for only a few seconds. Thus, the finitetime control for the guidance system is necessary in many practical guidance cases.
Since the 1990s, with the development of finite-time stability theories [11-14], the study on the finite-time-convergent (FTC) control method has increasingly became a research hotspot. Some guidance laws based on FTC control have been developed. The most representative one is the FTCG law proposed in [15]. The authors in [16, 17] adopted terminalsliding-mode method to design FTCG laws. However, in order to guarantee the stability of guidance system under the condition of maneuvering target, the FTCG laws in [15-17] contain a discontinuous control term, which brings undesirable chattering phenomenon.

It is well known that the chattering phenomenon may reduce the performance of system and cause the instability of whole system. Thus, research on chatter-free FTCG laws has the important practical and theoretical significance. In [15], to alleviate the chattering phenomenon, a saturation function was utilized to replace the symbolic function of the guidance input. However, to do this, the disturbance rejection performance is sacrificed. To alleviate the chattering phenomenon and hold the disturbance rejection performance, some FTCG laws were designed in [18], by employing a non-smooth disturbance observer (NSDOB) to estimate the 
target acceleration. The FTCG law in [18] eliminates the effect of target acceleration without using the saturation function and the symbolic function. Thus, the chattering problem is eliminated in [18].

However, for the above-mentioned NSDOB-based FTCG laws in [18], the upper bound of derivative of target acceleration must be known. In reality, the maneuvering characteristic of the target is complex; thus it is difficult to know the upper bound in advance. So far, fewer FTCG laws have been developed in the absence of the upper bound. It is well known that the extended state observer (ESO) is a powerful DO. Compared with NSDOB used in [18], ESO requires no information on the target acceleration. In [19], the target acceleration was estimated by ESO, and then a FTCG law was designed without using the upper bound of derivative of target acceleration.

The ESO-based FTCG (ESOFTCG) laws in [19], however, still have limitation: ESO cannot guarantee the estimation error fully converges to zero when the target acceleration is time-varying. Thus, ESOFTCG law only can guarantee the sliding surface converges to a neighbourhood of zero. In particular, if the target acceleration is fast varying, the estimation error is very large. And then the finite-time convergent feature of ESOFTCG law in [19] may be destroyed by the large estimation error of ESO. Actually, most of the target acceleration instances are time-varying in practical engineering. Thus, the ESOFTCG law in [19] may not work well in the practical situations.

In this paper, a new adaptive finite-time disturbance observer (AFDO) based on ASTW is proposed to estimate the time-varying target acceleration. And then a novel FTCG law is designed based on the output of AFDO. The main contributions of this paper lie in the following aspects:

(1) In the absence of the upper bound information of time-varying target acceleration, the proposed AFDO can fully estimate the target acceleration. Compared with NSDOB used in [18], AFDO requires no a priori information on the target acceleration. Unlike ESO used in [19], the advantage of AFDO is that the estimation error of AFDO can fully converge to zero in finite time when the target acceleration is timevarying.

(2) The proposed FTCG law is strictly finite-time convergent in the presence of time-varying target acceleration. When the target acceleration is time-varying, the proposed guidance law can strictly guarantee the sliding surface converges to zero in finite time rather than a neighbourhood of zero in [19]. Moreover, with the help of AFDO, the proposed FTCG law does not require the a priori information on the target acceleration which is needed in [18].

The remaining parts of this paper are as follows. In Section 2, the guidance model, the design objective, and the design idea of this paper are expounded. The main results are presented in Section 3. In Section 3, a novel observer AFDO is developed and the stability proof of the estimation with AFDO is presented. Then, a AFDO-based FTCG law

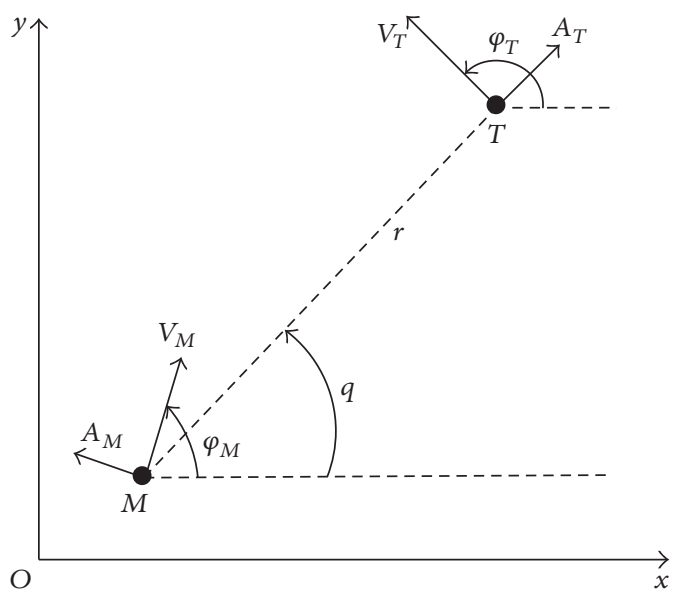

FIGURE 1: Interception geometry.

is proposed, and the finite-time stability of the law is also obtained. In Section 4, a simulation verifies the effectiveness of both AFDO and the proposed FTCG law. In Section 5, the conclusion of the whole paper is presented.

Notations. The following notations will be used in this paper: $t_{0}$ denotes the initial time. Let $\|\cdot\|$ denote the Euclidean norm of a vector and its induced norm of a matrix.

\section{Problem Formulation}

Consider a standard 2D dimensional geometry of interception shown in Figure 1. The origin $M$ is the missile and $T$ the target. The positions of the missile are $x_{M}$ and $y_{M}$. The positions of the target are $x_{T}$ and $y_{T} \cdot q$ is the LOS angle, $r$ is the range along LOS, $A_{M}$ and $A_{T}$ are the normal acceleration instances of the missile and target, and $V_{M}$ and $V_{T}$ are the velocities of missile and target. $\varphi_{m}$ and $\varphi_{T}$ are the flight path angles of missile and target. The relative motion between the missile and its target can be expressed by the following equations [19]:

$$
\begin{aligned}
\dot{r} & =V_{T} \cos \left(q-\varphi_{T}\right)-V_{M} \cos \left(q-\varphi_{M}\right), \\
r \dot{q} & =-V_{T} \sin \left(q-\varphi_{T}\right)+V_{M} \sin \left(q-\varphi_{M}\right), \\
\dot{\varphi}_{M} & =\frac{A_{M}}{V_{M}}, \\
\dot{\varphi}_{T} & =\frac{A_{T}}{V_{T}} .
\end{aligned}
$$

Let $V_{r}=V_{T} \cos \left(q-\varphi_{T}\right)-V_{M} \cos \left(q-\varphi_{M}\right), V_{\lambda}=-V_{T} \sin (q-$ $\left.\varphi_{T}\right)+V_{M} \sin \left(q-\varphi_{M}\right), A_{T r}=A_{T} \sin \left(q-\varphi_{T}\right)$, and $A_{T \lambda}=$ $A_{T} \cos \left(q-\varphi_{T}\right)$. Differentiating (1) yields [19]

$$
\begin{aligned}
\dot{q} & =\frac{V_{\lambda}}{r}, \\
\dot{V}_{\lambda} & =-\frac{V_{r} V_{\lambda}}{r}+A_{T \lambda}-A_{M} \cos \left(q-\varphi_{M}\right) .
\end{aligned}
$$


To achieve the hit-to-kill interception, a direct interception guidance strategy is given as [19]

$$
V_{\lambda} \longrightarrow c_{0} \sqrt{r}
$$

where $c_{0}$ is a constant. Thus for the guidance strategy (3), the guidance error is $V_{\lambda}-c_{0} \sqrt{r}$. In order to satisfy condition (3), the sliding surface is chosen as [19]

$$
S=V_{\lambda}-c_{0} \sqrt{r} .
$$

If $S=0$ can be satisfied in finite time, the objective of FTCG will be achieved [19].

Using the method in [16], $S=0$ can be satisfied in finite time by the following CFTCG law:

$$
\begin{aligned}
A_{M} & =\frac{1}{\cos \left(q-\varphi_{M}\right)}\left(\tau S+\sigma|S|^{\gamma} \operatorname{sign}(S)-\frac{V_{r} V_{\lambda}}{r}\right. \\
& \left.-\frac{\left(c_{0} / 2\right) V_{r}}{\sqrt{r}}+d \operatorname{sign}(S)\right),
\end{aligned}
$$

where $\tau>0, \sigma>0,0<\gamma<1$. $\operatorname{sign}(\cdot)$ represents the sign function, and its expression can be found in [16]. From [16], it can be known that $\sigma|S|^{\gamma} \operatorname{sign}(S)$ play a very important role in the CFTCG law (5). The convergence time of the sliding surface $S$ decreases as the value of $\sigma$ is increased and the value of $\gamma$ is decreased. From [16], it also can be known that the finite-time convergent feature of CFTCG law may be destroyed if the target acceleration $A_{T \lambda}$ is not equal to zero. Thus $d \operatorname{sign}(S)$ in (5) is used to eliminate the effect of target acceleration. And the constant $d$ must be selected as the upper bound of the target acceleration:

$$
\left|A_{T \lambda}\right| \leq d .
$$

However, $d \operatorname{sign}(S)$ is discontinuous and brings chattering problem. To avoid the chattering, NSDOB is used to estimate the target acceleration in [18], a non-smooth-control-based finite-time convergent guidance (NSCFTCG) law can be designed by using the method in [18]:

$$
\begin{aligned}
A_{M} & =\frac{1}{\cos \left(q-\varphi_{M}\right)}\left(\tau_{2} S+\sigma_{2}|S|^{\gamma_{2}} \operatorname{sgn}(S)-\frac{V_{r} V_{\lambda}}{r}\right. \\
& \left.-\frac{\left(c_{0} / 2\right) V_{r}}{\sqrt{r}}+p_{1}\right),
\end{aligned}
$$

where $p_{1}$ is the estimation of $A_{T \lambda}$ and given by following nonsmooth disturbance observer (NSDOB):

$$
\begin{aligned}
& \dot{p}_{0}=-\frac{V_{r} V_{\lambda}}{r}-A_{M} \cos \left(q-\varphi_{M}\right)+v_{0}, \\
& v_{0}=-\lambda_{p 0}\left|p_{0}-V_{\lambda}\right|^{2 / 3} \operatorname{sign}\left(p_{0}-V_{\lambda}\right)+p_{1}, \\
& \dot{p}_{1}=v_{1}, \\
& v_{1}=-\lambda_{p 1}\left|p_{1}-v_{0}\right|^{1 / 2} \operatorname{sign}\left(p_{1}-v_{0}\right)+p_{2}, \\
& \dot{p}_{2}=-\lambda_{p 2} \operatorname{sign}\left(p_{2}-v_{1}\right),
\end{aligned}
$$

where $\lambda_{p 0}=2 L^{1 / 3}, \lambda_{p 1}=1.5 L^{1 / 2}$, and $\lambda_{p 2}=1.1 L$. $L$ must be selected as the upper bound of $\dot{A}_{T \lambda}$.

$$
\left|\dot{A}_{T \lambda}\right| \leq L \text {. }
$$

However, the upper bound information $d$ and $L$ may not be easily obtained because the maneuvering characteristic of the target is complex. If $d$ and $L$ are unknown, the CFTCG law (5) and NSCFTCG law (7) are no longer available. To avoid using the upper bound information and eliminate the chattering problem, ESO is used to estimate the target acceleration in [19]. The guidance laws (5) and (7) are modified as the following ESOFTCG [19]:

$$
\begin{aligned}
A_{M} & =\frac{1}{\cos \left(q-\varphi_{M}\right)}\left(\tau S+\sigma|S|^{\gamma} \operatorname{sign}(S)-\frac{V_{r} V_{\lambda}}{r}\right. \\
& \left.-\frac{\left(c_{0} / 2\right) V_{r}}{\sqrt{r}}+Z_{2}\right),
\end{aligned}
$$

where the estimation of target acceleration $Z_{2}$ is given by following ESO:

$$
\begin{aligned}
E_{1}= & Z_{1}-V_{\lambda}, \\
\dot{Z}_{1}= & Z_{2}-\beta_{01} E_{1}-\frac{V_{r} V_{\lambda}}{r} \\
& -A_{M} \cos \left(q-\varphi_{M}\right), \\
\dot{Z}_{2}= & -\beta_{02} \operatorname{fal}\left(E_{1}, \alpha_{1}, \delta\right), \\
\operatorname{fal}\left(E_{1}, \alpha_{1}, \delta\right)= & \begin{cases}\left|E_{1}\right|^{\alpha_{1}} \operatorname{sign}\left(E_{1}\right), & \left|E_{1}\right|>\delta, \\
\frac{E_{1}}{\delta^{1-\alpha_{1}}}, & \text { otherwise. }\end{cases}
\end{aligned}
$$

From [19], it can be known that the ESOFTCG law (10) can drive the trajectory of the closed-loop system (2) into a neighbourhood of the sliding surface in finite time:

$$
\begin{aligned}
& \lim _{\theta \rightarrow \theta_{0}} S \in\left(S^{\gamma} \leq \frac{\left|E_{2}\right|}{(1-\theta) \sigma}\right), \\
& \left|E_{2}\right|= \begin{cases}\beta_{01}\left|\frac{g(t)}{\beta_{02}}\right|^{1 / \alpha_{1}}, & \text { if }\left|E_{1}\right|>\delta, \\
\frac{\beta_{01}\left|g(t) \delta^{1-\alpha_{1}}\right|}{\beta_{02}}, & \text { if }\left|E_{1}\right| \leq \delta,\end{cases}
\end{aligned}
$$

where $E_{2}$ is the estimation error of ESO (11) and satisfied $E_{2}=Z_{2}-A_{T \lambda}$. And $g(t)$ is the varying rate of the target acceleration; that is, $g(t)=\dot{A}_{T \lambda} \cdot \beta_{01}, \beta_{02}, \alpha_{1}$, and $\delta$ are constant.

Motivation of This Paper. Unlike the CFTCG law (5) and NSCFTCG law (7), the ESOFTCG law (10) does not need the upper bounds $d$ and $L$. However, from (13), it is clear that ESOFTCG law (10) cannot strictly guarantee the sliding surface $S$ converges to zero in finite time if the varying rate of the target acceleration $g(t) \neq 0$. Moreover, the upper bound 
of $|S|$ will increase progressively as $|g(t)|$ become bigger. Thus the requirement of FTCG cannot be guaranteed by the FTCG law (10) if the varying rate $g(t)$ is very large (in Section 4 of this paper, the simulation result also demonstrates that the performance of existing ESOFTCG law is poor when the target acceleration is fast time-varying). This motivates the research topic of this paper, that is, for the missile in the presence of time-varying target acceleration, designing a new FTCG law to strictly guarantee $S$ converge to zero in finite time without using the upper bounds $d$ and $L$.

Like [19], the following assumption should be assumed to be valid throughout this paper.

Assumption 1. The target acceleration $A_{T \lambda}$ is unknown bounded disturbance and satisfied the following condition:

$$
\begin{aligned}
& \left|A_{T \lambda}\right| \leq A_{T \lambda}^{\max }, \\
& \left|\dot{A}_{T \lambda}\right| \leq \dot{A}_{T \lambda}^{\max },
\end{aligned}
$$

where $A_{T \lambda}^{\max }$ and $\dot{A}_{T \lambda}^{\max }$ are unknown positive constant.

Assumption 1 implies that it is unnecessary to know the upper bound information $d$ of (6) and $L$ of (9).

\section{Main Result}

3.1. Observer Design and Stability Analysis. In this section, a new adaptive finite-time disturbance observer (AFDO) will be proposed to estimate the target acceleration based on ASTW algorithm. The performance of AFDO will not be affected by the varying rate of target acceleration. And AFDO requires no a priori information on the target acceleration.

Before giving the AFDO and guidance law of this paper, the following Lemmas 2 and 3, which play important role in the subsequent analysis, are recalled here for convenience.

Lemma 2 (see [14]). Provided that $V(x, t)$ is a differentiable and nonnegative scalar function, and $V(x, t)$ satisfies the differential inequality $\dot{V}(x, t) \leq-\alpha V^{\gamma}(x, t)$, where $\alpha>0$ and $0<\gamma<1, \alpha$ and $\gamma$ are constants, then we have

$$
V(x, t)=0, \quad \forall t>t_{r},
$$

where $t_{r} \leq t_{0}+V^{1-\gamma}\left(x_{0}, t_{0}\right) / \alpha(1-\gamma), V\left(x_{0}, t_{0}\right)$ is the initial value and $t_{0}$ is the initial time, and $V\left(x_{0}, t_{0}\right)$ is bounded.

The ASTW algorithm is given by the following lemma.

Lemma 3 (ASTW algorithm [20]). Consider the following differential inclusion:

$$
\begin{aligned}
& \dot{e}=-\lambda(t)|e|^{1 / 2} \operatorname{sign}(e)+\varphi, \\
& \dot{\varphi}=-k(t) \operatorname{sign}(e)+\dot{v},
\end{aligned}
$$

where $\lambda(t)$ and $k(t)$ are given as

$$
\begin{aligned}
& \lambda(t)=2 \sqrt{l(t)}, \\
& k(t)=4 l(t),
\end{aligned}
$$

where $l(t)$ is a positive time-varying scalar. The adaptive law of $l(t)$ is given by

$$
\dot{l}(t)= \begin{cases}\bar{q}, & \text { if } e \neq 0, \\ 0, & \text { otherwise }\end{cases}
$$

where $\bar{q}$ is a positive constant. If the following condition can be satisfied,

$$
|\dot{v}| \leq \bar{c}
$$

where $\bar{c}$ is an unknown constant, then e and $\dot{e}$ will converge to zero in finite time.

Other similar ASTW algorithms can be seen in [21-24]. Then AFDO and the stability analysis are given in Theorem 4 .

Theorem 4. Taking guidance system (2) into consideration, the AFDO (20) is constructed.

$$
\begin{aligned}
H_{1}= & V_{\lambda}-V_{1}, \\
\dot{V}_{1}= & \lambda_{1}\left|H_{1}\right|^{1 / 2} \operatorname{sign}\left(H_{1}\right)+J_{1}-\frac{V_{r} V_{\lambda}}{r} \\
& -A_{M} \cos \left(q-\varphi_{M}\right), \\
\dot{J}_{1}= & k_{1} \operatorname{sign}\left(H_{1}\right), \\
\lambda_{1}= & 2 \sqrt{l_{1}}, \\
k_{1}= & 4 l_{1} .
\end{aligned}
$$

The adaptive law of $l_{1}$ is given by

$$
\dot{l}_{1}= \begin{cases}\bar{q}_{1}, & \text { if } H_{1} \neq 0, \\ 0, & \text { otherwise }\end{cases}
$$

where $l_{1}\left(t_{0}\right)$ and $\bar{q}_{1}$ are positive constants. Assumption 1 is valid. The estimation error of AFDO is defined as $\mathrm{H}_{2}=J_{1}-A_{T \lambda}$. Then the estimation error $\mathrm{H}_{2}$ will converge to zero in finite time.

Proof. Differentiating $H_{1}$ gives

$$
\dot{H}_{1}=\dot{V}_{\lambda}-\dot{V}_{1} \text {. }
$$

Substituting the expression of $\dot{V}_{1}$ in (20) and $\dot{V}_{\lambda}$ in (2) into (22) yields

$$
\begin{aligned}
\dot{H}_{1} & =-\frac{V_{r} V_{\lambda}}{r}+A_{T \lambda}-A_{M} \cos \left(q-\varphi_{M}\right) \\
& -\left(\lambda_{1}\left|H_{1}\right|^{1 / 2} \operatorname{sign}\left(H_{1}\right)+J_{1}-\frac{V_{r} V_{\lambda}}{r}\right. \\
& \left.-A_{M} \cos \left(q-\varphi_{M}\right)\right)=-\lambda_{1}\left|H_{1}\right|^{1 / 2} \operatorname{sign}\left(H_{1}\right)-J_{1} \\
& +A_{T \lambda} .
\end{aligned}
$$

Let $\varphi_{1}=-J_{1}+A_{T \lambda}$. Differentiating $\varphi_{1}$ gives

$$
\dot{\varphi}_{1}=-\dot{J}_{1}+\dot{A}_{T \lambda} .
$$


Substituting the expression of $\dot{J}_{1}$ in (20) into (24) yields

$$
\dot{\varphi}_{1}=-k_{1} \operatorname{sign}\left(H_{1}\right)+\dot{A}_{T \lambda}
$$

Combining (23) and (25), we have

$$
\begin{aligned}
& \dot{H}_{1}=-\lambda_{1}\left|H_{1}\right|^{1 / 2} \operatorname{sign}\left(H_{1}\right)+\varphi_{1}, \\
& \dot{\varphi}_{1}=-k_{1} \operatorname{sign}\left(H_{1}\right)+\dot{A}_{T \lambda},
\end{aligned}
$$

where

$$
\begin{aligned}
& \lambda_{1}=2 \sqrt{l_{1}} \\
& k_{1}=4 l_{1} \\
& \dot{l}_{1}= \begin{cases}\bar{q}_{1}, & \text { if } H_{1} \neq 0 \\
0, & \text { otherwise }\end{cases}
\end{aligned}
$$

It is not difficult to note that (26), (27), and (28) have the same structure as (16), (17), and (18) in Lemma 3. Moreover, since Assumption 1 is valid, condition (19) can be satisfied. Then, according to Lemma 3, the following equations can be satisfied:

$$
\begin{aligned}
& H_{1}=0, \\
& \dot{H}_{1}=0,
\end{aligned}
$$

$$
\forall t \geq t_{1}
$$

where $t_{1}$ is a finite time.

Substituting (29) into (23), we have

$$
A_{T \lambda}=J_{1}, \quad \forall t \geq t_{1} .
$$

Then it is clear that the estimation error $H_{2}=J_{1}-A_{T \lambda}$ will converge to zero in finite time $t_{1}$. The demonstration of Theorem 4 is completed.

In order to implement the AFDO (20), the following assumption is needed.

\section{Assumption 5. $r, q, \dot{r}, \dot{q}, A_{M}$, and $\varphi_{M}$ are measurable.}

Remark 6. Note that the third formula $J_{1}$ in (20) is most important. Theorem 4 shows that $J_{1}$ can estimate the target acceleration $A_{T \lambda}$. Unlike $Z_{2}$ in ESO (11), which only can estimate the target acceleration with the estimation error $E_{2}$, $J_{1}$ can fully estimate the target acceleration in finite time. And like ESO (11), AFDO (20) do not need the upper bound of $A_{T \lambda}$ and $\dot{A}_{T \lambda}$, which only need $A_{T \lambda}$ and $\dot{A}_{T \lambda}$ to be bounded.

3.2. Guidance Law Design and Stability Analysis. After estimating the target acceleration with AFDO, a novel FTCG law is designed as follows:

$$
\begin{aligned}
A_{M} & =\frac{1}{\cos \left(q-\varphi_{M}\right)}\left(\tau_{1} S+\sigma_{1}|S|^{\gamma_{1}} \operatorname{sign}(S)-\frac{V_{r} V_{\lambda}}{r}\right. \\
& \left.-\frac{\left(c_{0} / 2\right) V_{r}}{\sqrt{r}}+J_{1}\right),
\end{aligned}
$$

where $J_{1}$ is given by the AFDO (20). $\tau_{1}>0, \sigma_{1}>0$, and $0<$ $\gamma_{1}<1$. Then Theorem 7 will prove the finite-time-convergent feature of the closed-loop system under the AFDO-based guidance law (31).

Theorem 7. Consider the guidance system (2) adopts AFDObased guidance law (31). If Assumption 1 is valid, then the trajectory of system (2) can be driven onto the sliding surface $(S=0)$ in finite time.

Proof. From (2) and (4), we have

$$
\begin{aligned}
\dot{S} & =\dot{V}_{\lambda}-\frac{\left(c_{0} / 2\right) V_{r}}{\sqrt{r}} \\
& =-\frac{V_{r} V_{\lambda}}{r}+A_{T \lambda}-A_{M} \cos \left(q-\varphi_{M}\right)-\frac{\left(c_{0} / 2\right) V_{r}}{\sqrt{r}} .
\end{aligned}
$$

Substituting the proposed AFDO-based guidance law (31) into (32), we have

$$
\dot{S}=-\tau_{1} S-\sigma_{1}|S|^{\gamma_{1}} \operatorname{sign}(S)+A_{T \lambda}-J_{1} .
$$

Construct Lyapunov function $V_{2}$ as

$$
V_{2}=\frac{1}{2} S^{2}
$$

Then calculating the time derivative of $V_{2}$ along the trajectories of (33), we get

$$
\begin{aligned}
\dot{V}_{2} & =S \dot{S}=S\left(-\tau_{1} S-\sigma_{1}|S|^{\gamma_{1}} \operatorname{sign}(S)-J_{1}+A_{T \lambda}\right) \\
& =-\tau_{1} S^{2}-\sigma_{1}|S|^{\gamma_{1}+1}-\left(J_{1}-A_{T \lambda}\right) S \\
& \leq-2^{\left(\gamma_{1}+1\right) / 2} \sigma_{1} V_{2}^{\left(\gamma_{1}+1\right) / 2}-\left(J_{1}-A_{T \lambda}\right) S \\
& \leq-2^{\left(\gamma_{1}+1\right) / 2} \sigma_{1} V_{2}^{\left(\gamma_{1}+1\right) / 2}+\sqrt{2}\left|J_{1}-A_{T \lambda}\right| V_{2}^{1 / 2} .
\end{aligned}
$$

From (35), it denotes that $V_{2}$ is affected by the estimation error $H_{2}=J_{1}-A_{T \lambda}$. Thus, in the following, the proof of Theorem 7 consists of two steps. In the first step, it will be proved that $V_{2}$ will not escape to infinity before $\left(J_{1}-A_{T \lambda}\right)$ converges to zero. In the second step, it will be proved that $V_{2}$ will converge to zero in finite time after $\left(J_{1}-A_{T \lambda}\right)$ converges to zero. And the total convergence time of $V_{2}$ will be calculated.

Step 1. From (25) and Assumption 1, we have

$$
\left|\dot{\varphi}_{1}\right| \leq k_{1}+\left|\dot{A}_{T \lambda}\right| \leq k_{1}+\dot{A}_{T \lambda}^{\max } .
$$

From the expression of $k_{1}$ in (20), (36) can be rewritten as

$$
\left|\dot{\varphi}_{1}\right| \leq 4 l_{1}+\dot{A}_{T \lambda}^{\max } .
$$

Then considering (21) and (37), it can be deduced that $\left|\dot{\varphi}_{1}\right|$ is bounded by an unknown positive constant for $t \leq t_{1}$ :

$$
\left|\dot{\varphi}_{1}\right| \leq 4\left(\bar{q}_{1}\left(t_{1}-t_{0}\right)+l_{1}\left(t_{0}\right)\right)+\dot{A}_{T \lambda}^{\max }, \quad \forall t \leq t_{1} .
$$


From (38), we have

$$
\begin{aligned}
\left|\varphi_{1}\right| \leq & \left|\int_{t_{0}}^{t} \dot{\varphi}_{1} d t+\varphi_{1}\left(t_{0}\right)\right| \leq\left|\int_{t_{0}}^{t} \dot{\varphi}_{1} d t\right|+\left|\varphi_{1}\left(t_{0}\right)\right| \\
\leq & \int_{t_{0}}^{t}\left|\dot{\varphi}_{1}\right| d t+\left|\varphi_{1}\left(t_{0}\right)\right| \\
\leq & \left(4\left(\bar{q}_{1}\left(t_{1}-t_{0}\right)+l_{1}\left(t_{0}\right)\right)+\dot{A}_{T \lambda}^{\max }\right)\left(t-t_{0}\right) \\
& +\left|\varphi_{1}\left(t_{0}\right)\right| \\
\leq & \left(4\left(\bar{q}_{1}\left(t_{1}-t_{0}\right)+l_{1}\left(t_{0}\right)\right)+\dot{A}_{T \lambda}^{\max }\right)\left(t_{1}-t_{0}\right) \\
& +\left|J_{1}\left(t_{0}\right)\right|+\left|A_{T \lambda}\left(t_{0}\right)\right|, \quad \forall t \leq t_{1} .
\end{aligned}
$$

From (39), it is clear that $\varphi_{1}$ is bounded by unknown positive constant $\bar{c}_{2}$ in finite time $t_{1}$ :

$$
\left|\varphi_{1}\right|=\left|-J_{1}+A_{T \lambda}\right|=\left|J_{1}-A_{T \lambda}\right| \leq \bar{c}_{2}, \quad \forall t \leq t_{1},
$$

where $\bar{c}_{2}=\left(4\left(\bar{q}_{1}\left(t_{1}-t_{0}\right)+l_{1}\left(t_{0}\right)\right)+\dot{A}_{T \lambda}^{\max }\right)\left(t_{1}-t_{0}\right)+\left|J_{1}\left(t_{0}\right)\right|+$ $\left|A_{T \lambda}\left(t_{0}\right)\right|$ and $t_{1}$ is the convergence time of AFDO (20) and given in Theorem 4 .

Combining (40) with (35), we have

$$
\begin{aligned}
\dot{V}_{2} & \leq-2^{\left(\gamma_{1}+1\right) / 2} \sigma_{1} V_{2}^{\left(\gamma_{1}+1\right) / 2}+\sqrt{2}\left|J_{1}-A_{T \lambda}\right| V_{2}^{1 / 2} \\
& \leq\left(-2^{\left(\gamma_{1}+1\right) / 2} \sigma_{1} V_{2}^{\gamma_{1} / 2}+\sqrt{2} \bar{c}_{2}\right) V_{2}^{1 / 2}, \quad \forall t \leq t_{1} .
\end{aligned}
$$

From (41), it is clear that $\dot{V}_{2} \leq 0$ if $V_{2} \geq\left(\bar{c}_{2} / \sigma_{1} 2^{\gamma_{1} / 2}\right)^{2 / \gamma_{1}}$. Thus, it can be known that $V_{2}$ is bounded in finite time $t_{1}$ :

$$
V_{2} \leq \max \left\{\left(\frac{\bar{c}_{2}}{\sigma_{1} 2^{\gamma_{1} / 2}}\right)^{2 / \gamma_{1}}, V_{2}\left(t_{0}\right)\right\}, \quad \forall t \leq t_{1} .
$$

Thus $V_{2}$ will not escape to infinity before $\left(A_{T \lambda}+J_{1}\right)$ converges to zero; that is, $V_{2}\left(t_{1}\right)$ is bounded.

Step 2. Since Assumption 1 is valid, (30) will be satisfied in finite time $t_{1}$. Then combining (30) with (35), we have

$$
\dot{V}_{2} \leq-2^{\left(\gamma_{1}+1\right) / 2} \sigma_{1} V_{2}^{\left(\gamma_{1}+1\right) / 2}, \quad \forall t \geq t_{1} .
$$

$V_{2}\left(t_{1}\right)$ is bounded and has been proved in Step 1. As $V_{2}\left(t_{1}\right)$ is bounded, $\sigma_{1}>0$ and $0<\gamma_{1}<1$, and $V_{2}$ and $S$ will converge to zero in finite time $t_{2}$ based on Lemma 2:

$$
S=V_{2}=0, \quad \forall t \geq t_{2}
$$

The convergence time $t_{2}$ satisfies the following equation:

$$
t_{2} \leq \frac{V_{2}^{\left(1-\gamma_{1}\right) / 2}\left(t_{1}\right)}{2^{\left(1-\gamma_{1}\right) / 2} \sigma_{1}\left(1-\gamma_{1}\right)}+t_{1}
$$

The demonstration of Theorem 7 is completed.

Remark 8. From the result of Theorem 7, it is clear that the problem that ESOFTCG law (10) cannot strictly guarantee $S$ converge to zero is solved by the proposed AFDO-based FTCG law.
Remark 9. From Remark 4.3 in [19], it can be known that the boundary layer of the sliding surface in [19] is determined by the estimation error of the ESO. Thus, the parameter selection of the ESO is more important since it not only determines the performance of the ESO but also impacts the behavior of the sliding surface. However, in this paper, the estimation error of AFDO will converge to zero in finite time as soon as the parameters satisfy $l_{1}\left(t_{0}\right)>0$ and $\bar{q}_{1}>0$. Thus, the parameter selection in this paper is much simpler.

Remark 10. From [21], if the final miss distance is less than $0.25 \mathrm{~m}$, the hit-to-kill interception also can be satisfied.

Remark 11. Condition $H_{1}=0$ is difficult to be satisfied in practice due to numerical approximations and measurement noise. From [25], it can be known that the condition $H_{1}=0$ can be modified by the following dead-zone technique:

$$
\dot{l}_{1}= \begin{cases}\bar{q}_{1}, & \text { if }\left|H_{1}\right| \geq \eta \\ 0, & \text { otherwise }\end{cases}
$$

where $\eta$ is a sufficiently small positive value.

\section{Simulation Results}

This subsection shows the performances of the AFDO and the proposed AFDO-based guidance law. The initial positions of the missile are $x_{M}\left(t_{0}\right)=0$ and $y_{M}\left(t_{0}\right)=0$. The initial positions of the target are $x_{T}\left(t_{0}\right)=2000 \mathrm{~m}$ and $y_{T}\left(t_{0}\right)=$ $2000 \mathrm{~m}$. The initial path angles are $\varphi_{M}\left(t_{0}\right)=\pi / 4 \mathrm{rad}$ and $\varphi_{T}\left(t_{0}\right)=\pi / 3.8 \mathrm{rad}$. Seeker measurement delays for $30 \mathrm{~ms}$. In addition, the maximum limit of the missile acceleration command is selected as $200 \mathrm{~m} / \mathrm{s}^{2}$.

For the comparison, the ESOFTCG law (10) given in [19] are also considered in this section. The parameters of ESO (8) are chosen as $\beta_{01}=50, \beta_{02}=100, \alpha_{1}=0.2$, and $\delta=0.15$. The parameters of ESOFTCG law (10) are chosen as $\tau=10$, $\sigma=1$, and $\gamma=0.5$. Note that, in this paper, the parameters of ESOFTCG law and ESO are the same as those in [19] and used here to ensure the fairness of comparison.

The parameters of AFDO (20) are chosen as $\eta=0.0005$, $l_{1}\left(t_{0}\right)=5$, and $\bar{q}_{1}=1000$. The parameters of the proposed law (31) are chosen as $\tau_{1}=10, \sigma_{1}=1$, and $\gamma_{1}=0.5$.

Like [19], the parameter of the sliding surface $S$ in the ESOTFCG law and the proposed law is selected as $c_{0}=0.1$.

Case 1 (constant target acceleration). The target acceleration $A_{T \lambda}$ is given as

$$
A_{T \lambda}=-35 \mathrm{~m} / \mathrm{s}^{2}
$$

From (47), it can be known that the target acceleration is constant in Case 1. Figures 2(a)-2(d) and Table 1 show the simulation results for Case 1. From Figure 2(a), it is clear that the proposed law and the ESOFTCG law can guarantee the sliding surface converges to zero in finite time. Figure 2(b) shows that AFDO and ESO ensure the estimation error converges to zero. From Figure 2(c) and Table 1, it can be 

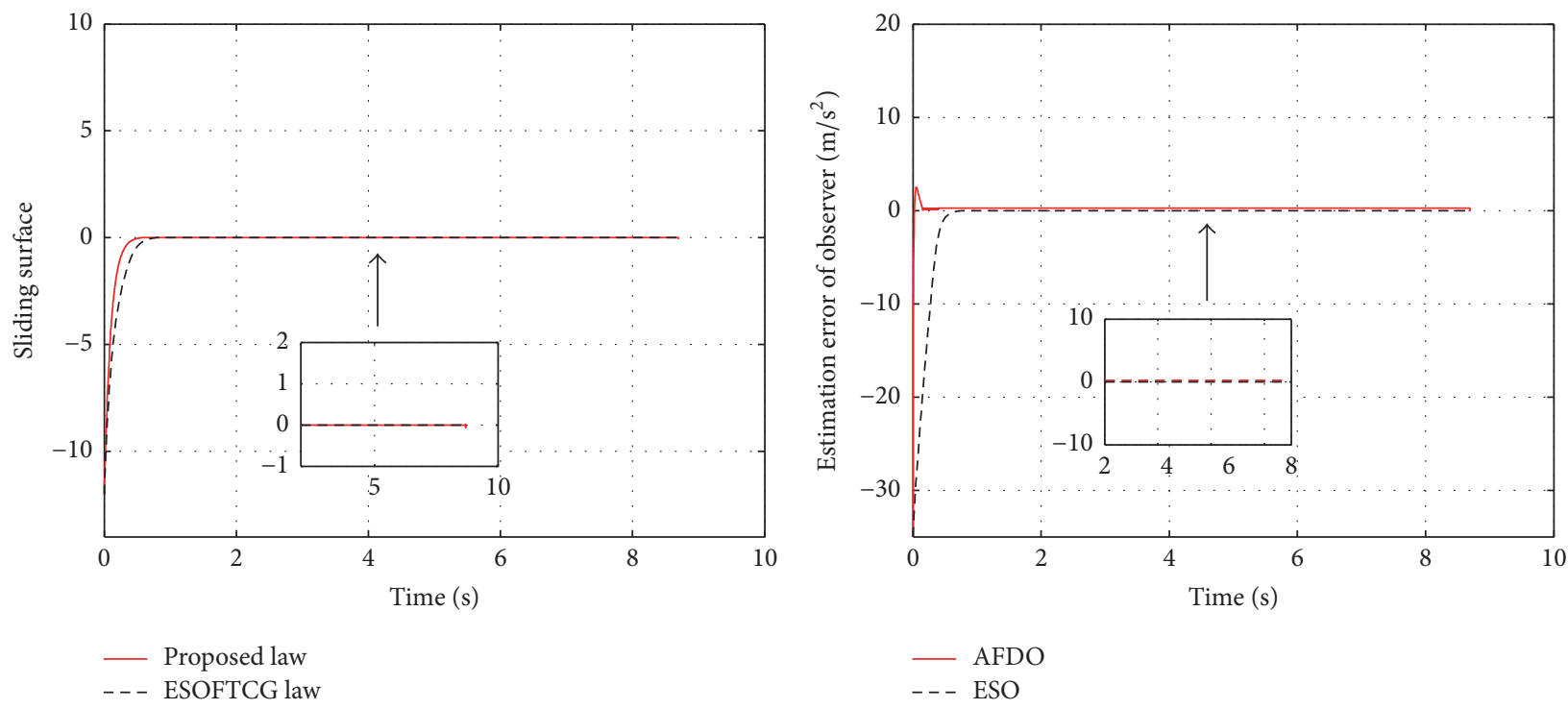

(a) Sliding surface

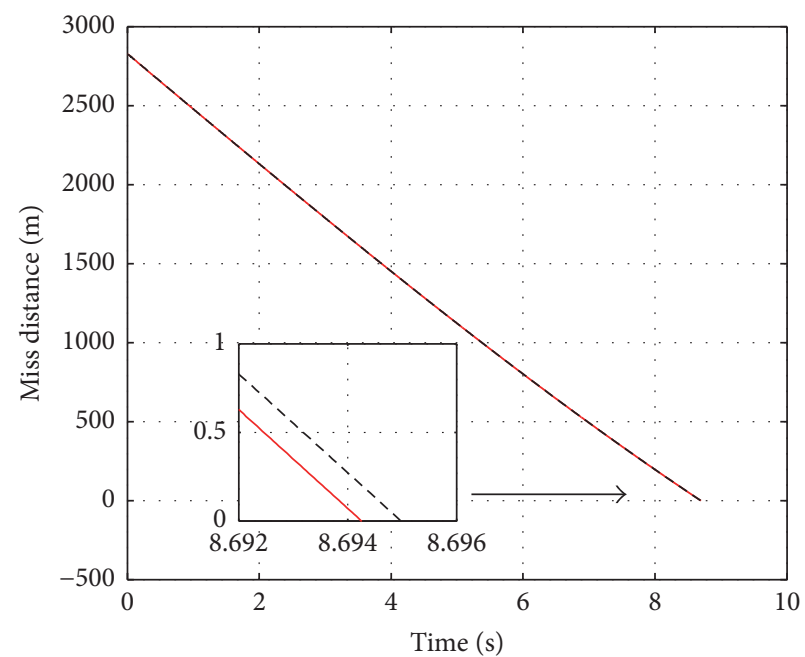

(b) Estimation error of observer

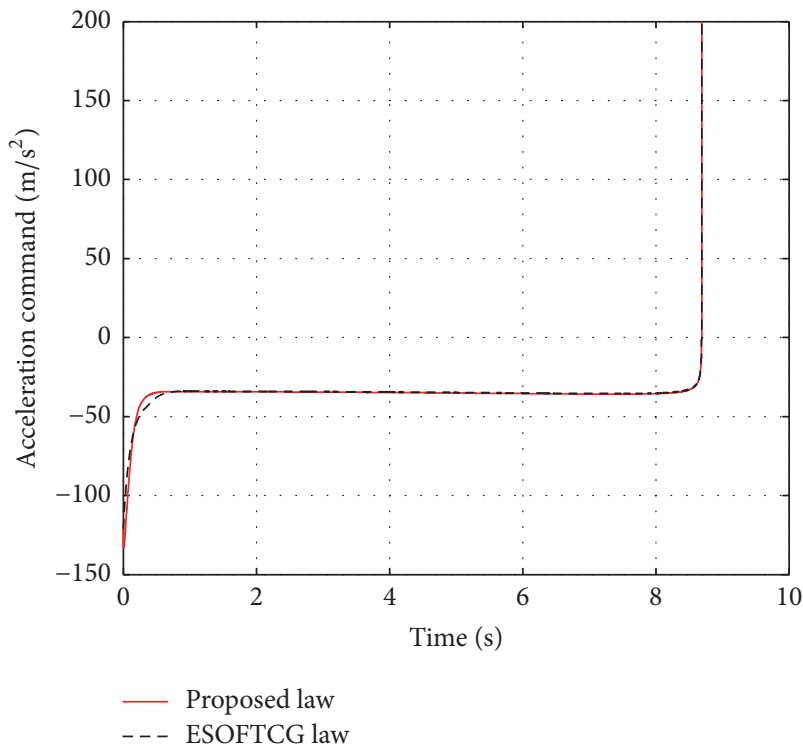

(c) Time history of miss distance

(d) Acceleration command

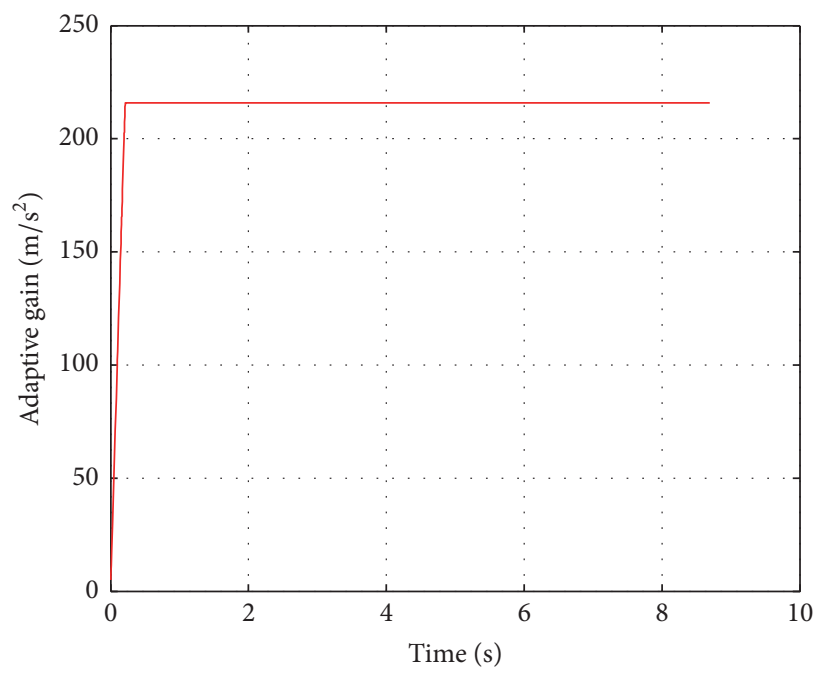

(e) Adaptive gain $l_{1}$

FiguRE 2: Simulation result for Case 1 (constant target acceleration). 
TABLE 1: Performance of guidance laws in Section 4.

\begin{tabular}{lcc}
\hline Case & Guidance law & Final miss distance $(\mathrm{m})$ \\
\hline \multirow{2}{*}{1} & Proposed law & 0.033 \\
& ESOFTCG law & 0.054 \\
\hline \multirow{2}{*}{3} & Proposed law & 0.079 \\
& ESOFTCG law & 0.237 \\
\hline \multirow{2}{*}{3} & Proposed law & 0.087 \\
& ESOFTCG law & 3.23 \\
\hline
\end{tabular}

known that the proposed law and ESOFTCG law guarantee the final miss distances are less than $0.1 \mathrm{~m}$, which means that the proposed law and ESOFTCG law can guarantee the missile accurately attacks the target (see Remark 10). From Figure 2(d), it is clear that the acceleration commands of the proposed law and ESOFTCG law are chatter-free. Thus the proposed method and method in [19] exhibit good performance in the presence of constant target acceleration.

Case 2 (slowly varying target acceleration). The target acceleration $A_{T \lambda}$ with a frequency $1 /(6 \pi) \mathrm{HZ}$ is given as

$$
A_{T \lambda}=175 \cos \left(\frac{t}{3}\right) \mathrm{m} / \mathrm{s}^{2}
$$

From (48), it can be known that the target acceleration is time-varying in Case 2. But the varying rate in Case 2 is small. Figures 3(a)-3(d) and Table 1 show the simulation results for Case 2. From Figure 3(a), it is clear that the proposed law guarantees the sliding surface converges to zero, but ESOFTCG law only can guarantee the sliding surface converges to a neighbourhood of zero. The reason for Figure 3(a) is that ESO cannot guarantee the estimation error converges to zero when the target acceleration is timevarying, while AFDO can guarantee the estimation error of AFDO converges to zero. The reason can be observed from Figure 3(b). Figure 3(c) and Table 1 show that that the proposed law and ESOFTCG law guarantee the final miss distances are less than $0.25 \mathrm{~m}$, which means that the proposed law and ESOFTCG law can accomplish hit-to-kill interception (see Remark 10). And, from Table 1, it also can be known that the proposed guidance law can achieve a smaller final miss distance.

Case 3 (fast varying target acceleration). The target acceleration $A_{T \lambda}$ with a frequency $2 / \pi \mathrm{HZ}$ is given as

$$
A_{T \lambda}=175 \cos (4 t) \mathrm{m} / \mathrm{s}^{2} .
$$

From (49), it can be known that the varying rate of target acceleration in Case 3 is much larger than that in Case 2. It is noted that the method in [19] does not consider the target acceleration of this type. Figures $4(\mathrm{a})-4(\mathrm{~d})$ and Table 1 show the simulation results for Case 3. From Figure 4(a), it is clear that the proposed law can guarantee the sliding surface converges to zero in finite time. But the ESOFTCG law cannot guarantee the sliding surface converges to zero. From Figure 4(b), it is clear that the AFDO can fully estimate the target acceleration, but the estimation error of ESO is very large. From Figure 4(c) and Table 1, it is clear that the proposed guidance law still can guarantee the final miss distance is less than $0.1 \mathrm{~m}$. Thus, the missile with the proposed law can accomplish hit-to-kill interception, while the final miss distance of ESOFTCG law is $3.2 \mathrm{~m}$, which means that the missile with ESOFTCG law cannot accomplish the hitto-kill interception (see Remark 10). The reason for Figures 4(a), 4(b), and 4(c) is that the estimation error of ESO is large when target acceleration is fast varying. And then the finite-time convergent feature of ESOFTCG law is destroyed by the large estimation error (the relationship between sliding surface, estimation error of ESO, and varying rate of target acceleration is shown in (13)). And the hit-to-kill guidance strategy cannot be accomplished by ESOFTCG law. Since AFDO can fully estimate target acceleration, the proposed law can still accomplish hit-to-kill interception in the present of the fast varying target acceleration.

According to the simulation results, the following can be concluded:

(1) AFDO can achieve a good estimation effect on the condition of the target acceleration instances with either low or high varying rate (Figures 2-4). But ESO can only have a good estimation effect on the condition of constant acceleration (Figure 2). If the target acceleration is varying, the estimation error of ESO will increase with the increase of the varying rate of target acceleration (Figures 3 and 4).

(2) The proposed guidance law can strictly guarantee the sliding surface converges to zero in finite time when the target acceleration is constant or time-varying (Figures 2-4). But ESOFTCG law only can guarantee the sliding surface converges to a neighbourhood of zero if the target acceleration is time-varying (Figures 3 and 4). In particular, if the target acceleration is fast varying, the estimation error of ESO is very large (Figure 4). Then the finite-time convergent feature of ESOFTCG law will be destroyed by the large estimation error of ESO (Figure 4).

(3) Unlike the CFTCG law, the proposed law does not need the upper bound of target acceleration.

(4) Since the target acceleration has been fully estimated by AFDO, the proposed guidance law has no discontinuous control term. Thus the chattering problem in CFTCG law is solved (Figures 2(d), 3(d), and 4(d)).

\section{Conclusion}

(1) In this paper, a novel adaptive finite-time disturbance observer (AFDO) based on ASTW algorithm was proposed, which does not need to know the upper bound of the target acceleration in advance. Moreover, the estimation error of AFDO strictly converges to zero in finite time even if the target acceleration is time-varying. 


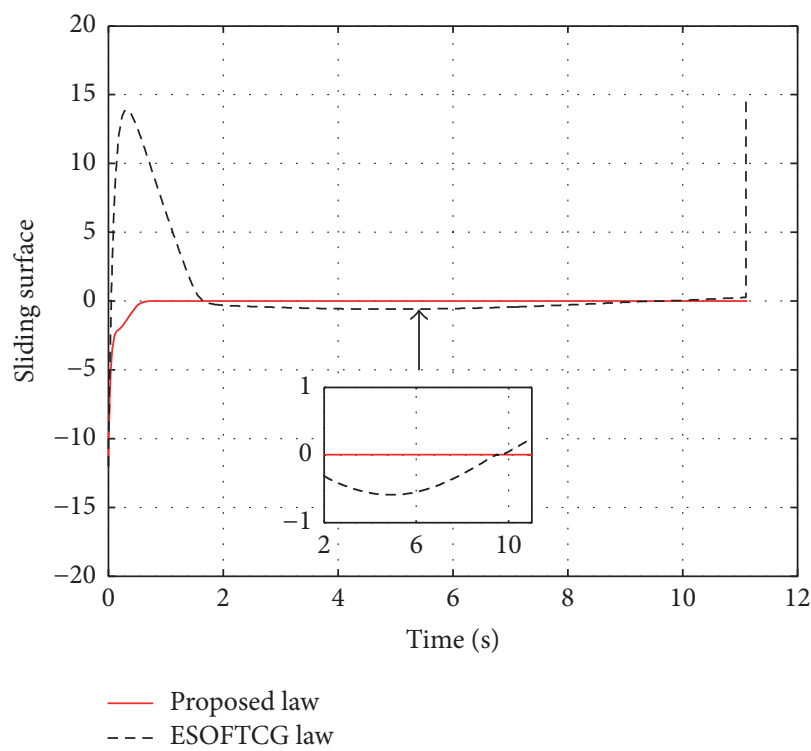

(a) Sliding surface

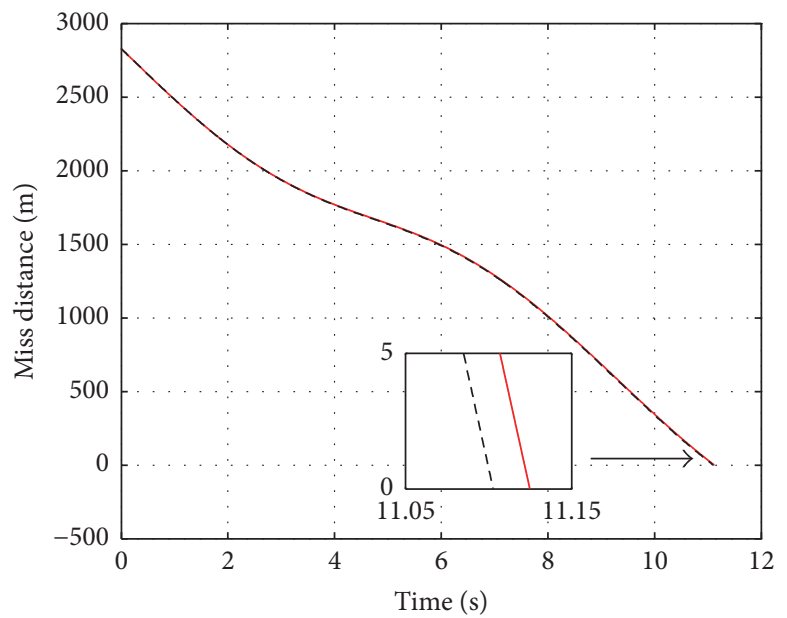

\section{- Proposed law}

- . - ESOFTCG law

(c) Time history of miss distance

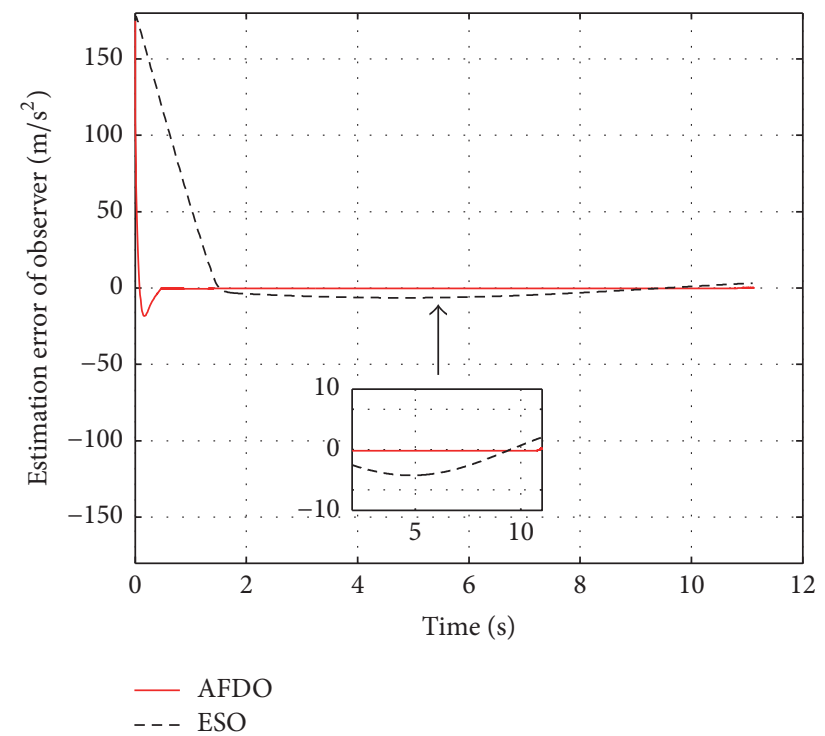

(b) Estimation error of observer

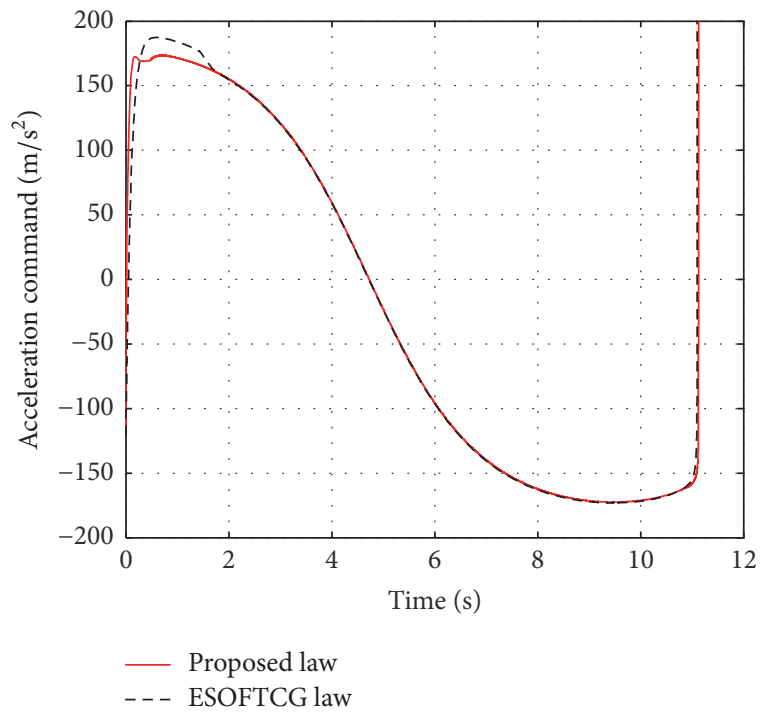

(d) Acceleration command

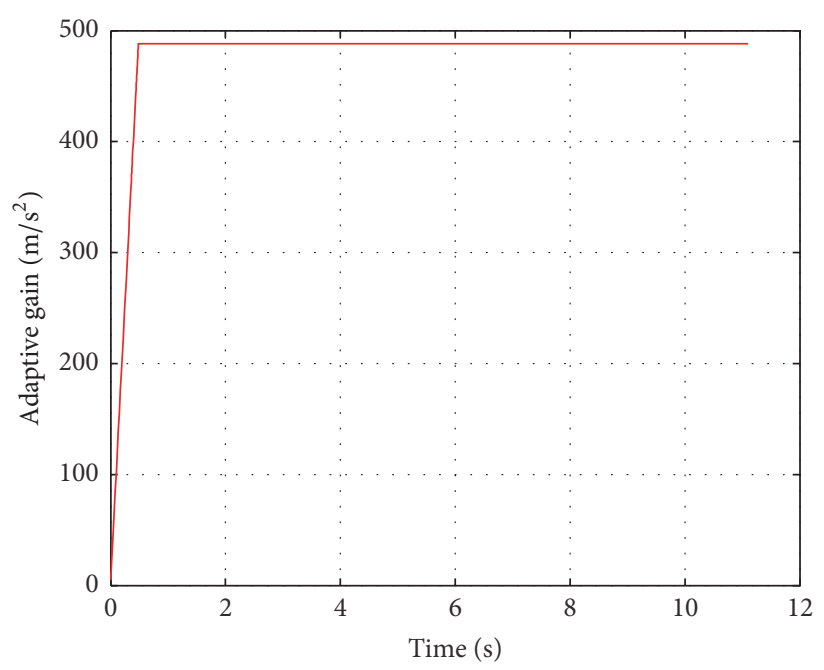

(e) Adaptive gain $l_{1}$

FIGURE 3: Simulation result for Case 2 (slowly varying target acceleration). 


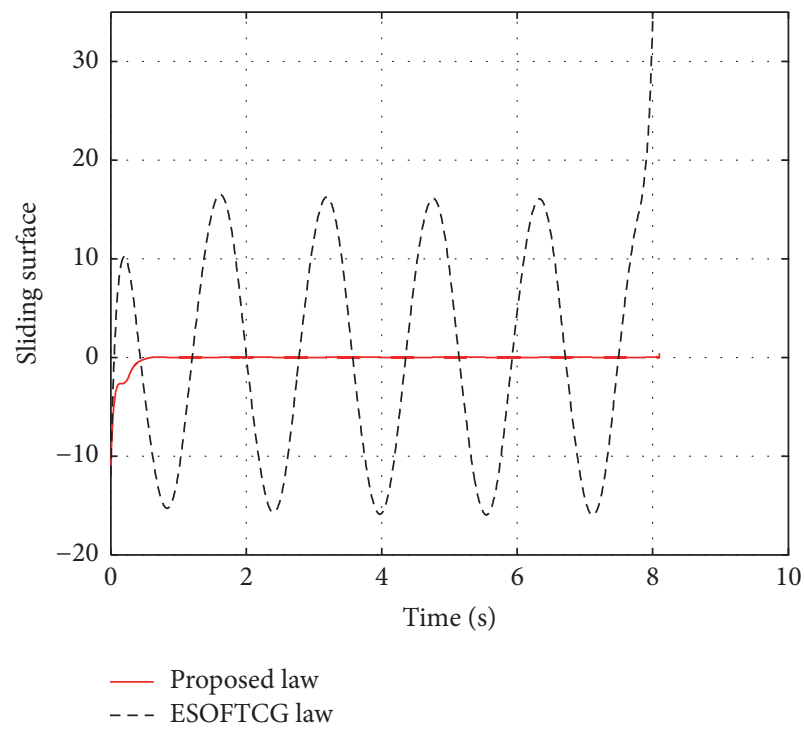

(a) Sliding surface

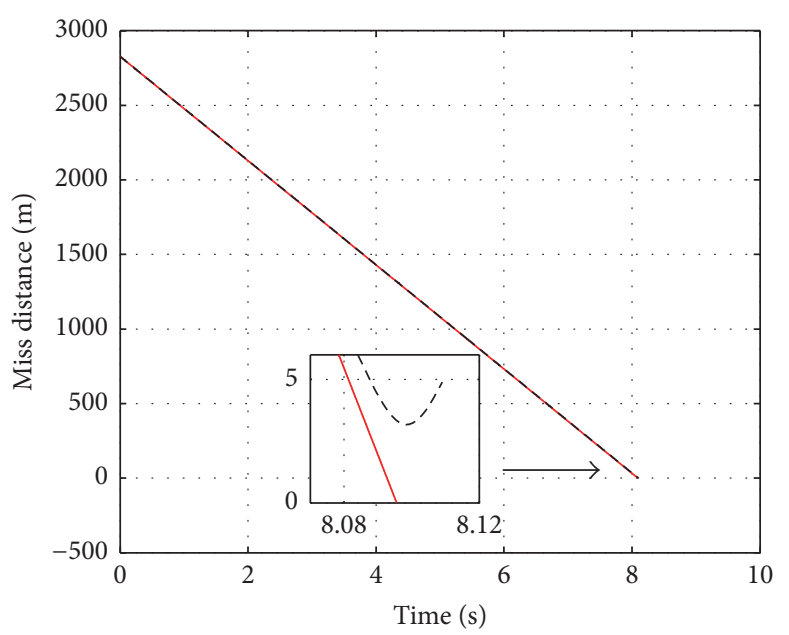

Proposed law

ESOFTCG law

(c) Time history of miss distance

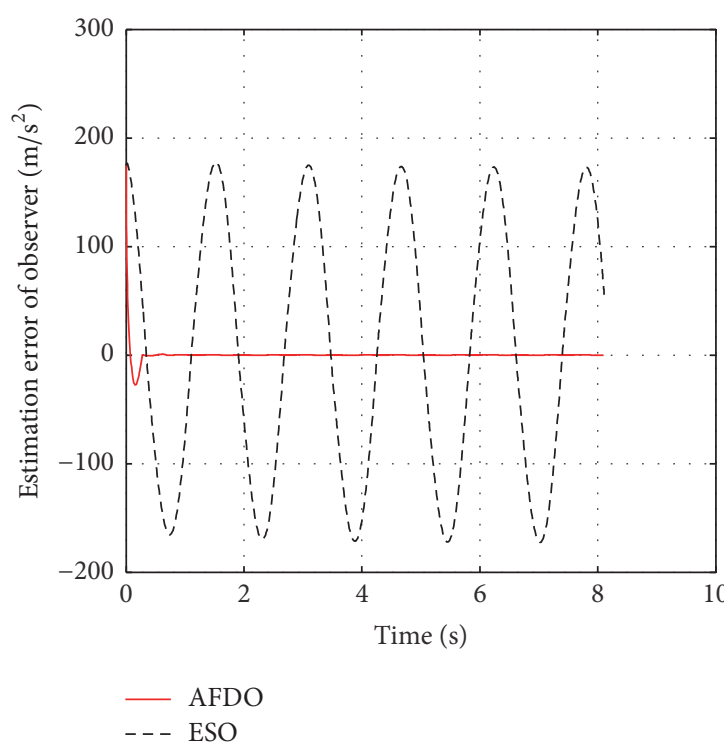

(b) Estimation error of observer

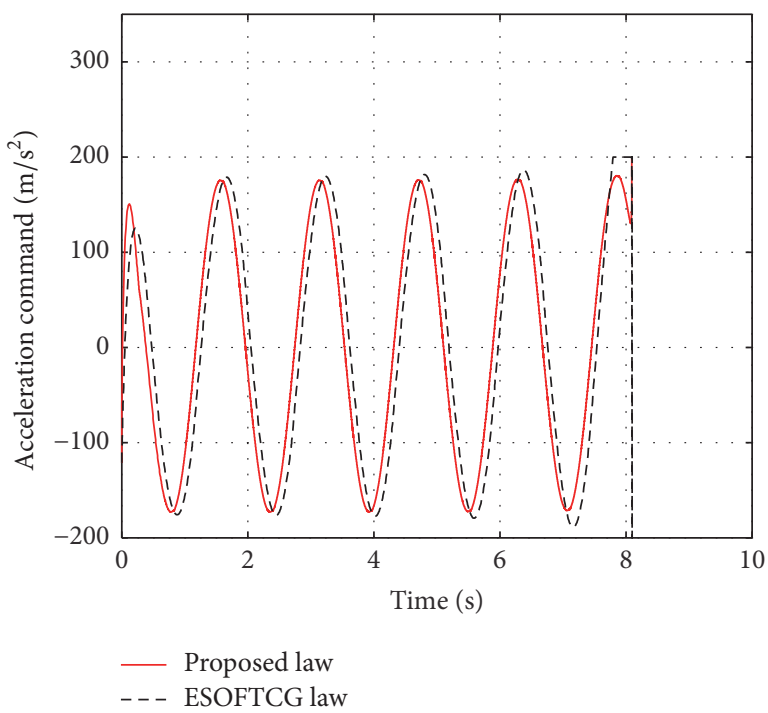

(d) Acceleration command

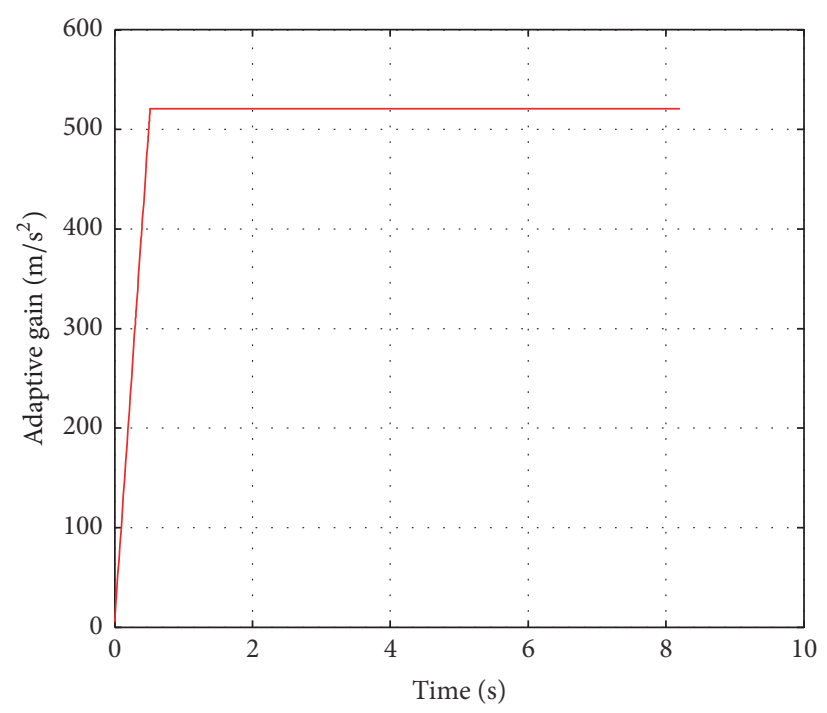

(e) Adaptive gain $l_{1}$

FIGURE 4: Simulation result for Case 3 (fast varying target acceleration). 
(2) Subsequently, a novel FTCG law based on AFDO was proposed. The newly proposed FTCG law has several advantages over existing FTCG laws. First, for the time-varying target acceleration, the proposed guidance law can strictly drive the trajectory of the closed-loop system onto the sliding-mode surface rather than a neighbourhood of sliding-mode surface in the ESOFTCG law. Second, unlike the CFTCG law, the proposed method requires no information on the target acceleration. Third, the chattering problem in the CFTCG law is completely avoided in this paper.

(3) Finally, mathematical simulation result demonstrated that the performances of the AFDO and the proposed guidance law are excellent.

\section{Competing Interests}

The authors declare that there is no conflict of interests regarding the publication of this paper.

\section{Acknowledgments}

This work is supported by the National Natural Science Foundation of China (61304238), the National High-Tech R\&D Program of China (2012AA120602, 2015AAxxx7033, and 2015AAxxx1008), and the Chinese Aerospace Supported Fund (2015-HT-XGD).

\section{References}

[1] S. N. Ghawghawe and D. Ghose, "Pure proportional navigation against time-varying target maneuvers," IEEE Transaction on Control Systems Technology, vol. 22, no. 4, pp. 589-594, 1999.

[2] M. Guelman, "The closed-form solution of true proportional navigation," IEEE Transactions on Aerospace and Electronic Systems, vol. AES-12, no. 4, pp. 472-482, 1976.

[3] P.-J. Yuan and J.-S. Chern, "Solutions of true proportional navigation for maneuvering and nonmaneuvering targets," Journal of Guidance, Control, and Dynamics, vol. 15, no. 1, pp. 268-271, 1992.

[4] C.-D. Yang, F.-B. Hsiao, and F.-B. Yeii, "Generalized guidance law for homing missiles," IEEE Transactions on Aerospace and Electronic Systems, vol. 25, no. 2, pp. 197-212, 1989.

[5] P.-J. Yuan and J.-S. Chern, "Ideal proportional navigation," Journal of Guidance, Control, and Dynamics, vol. 15, no. 5, pp. 1160-1165, 1992.

[6] S. N. Ghawghawe and D. Chose, "Pure proportional navigation against time-varying target maneuvers," IEEE Transactions on Aerospace and Electronic Systems, vol. 32, no. 4, pp. 1336-1347, 1996.

[7] C.-D. Yang and H.-Y. Chen, "Nonlinear Ho robust guidance law for homing missiles," Journal of Guidance, Control, and Dynamics, vol. 21, no. 6, pp. 882-890, 1998.

[8] D. Zhou, C. Mu, and T. Shen, "Robust guidance law with L2 gain performance," Transactions of the Japan Society for Aeronautical and Space Sciences, vol. 44, no. 144, pp. 82-88, 2001.

[9] N. Léchevin and C. A. Rabbath, "Lyapunov-based nonlinear missile guidance," Journal of Guidance, Control, and Dynamics, vol. 27, no. 6, pp. 1096-1102, 2004.
[10] S. D. Brierley and R. Longchamp, "Application of sliding-mode control to air-air interception problem," IEEE Transactions on Aerospace and Electronic Systems, vol. 26, no. 2, pp. 306-325, 1990.

[11] S. P. Bhat and D. S. Bernstein, "Finite-time stability of homogeneous systems," in Proceedings of the American Control Conference, pp. 2513-2514, Albuquerque, NM, USA, June 1997.

[12] S. P. Bhat and D. S. Bernstein, "Geometric homogeneity with applications to finite-time stability," Mathematics of Control, Signals, and Systems, vol. 17, no. 2, pp. 101-127, 2005.

[13] S. Ding, A. Levant, and S. Li, "Simple homogeneous slidingmode controller," Automatica. A Journal of IFAC, the International Federation of Automatic Control, vol. 67, pp. 22-32, 2016.

[14] S. P. Bhat and D. S. Bernstein, "Finite-time stability of continuous autonomous systems," SIAM Journal on Control and Optimization, vol. 38, no. 3, pp. 751-766, 2000.

[15] D. Zhou, S. Sun, and K. L. Teo, "Guidance laws with finite time convergence," Journal of Guidance, Control, and Dynamics, vol. 32, no. 6, pp. 1838-1846, 2009.

[16] Y. Zhang, M. Sun, and Z. Chen, "Finite-time convergent guidance law with impact angle constraint based on slidingmode control," Nonlinear Dynamics. An International Journal of Nonlinear Dynamics and Chaos in Engineering Systems, vol. 70, no. 1, pp. 619-625, 2012.

[17] S. R. Kumar, S. Rao, and D. Ghose, "Nonsingular terminal sliding mode guidance with impact angle constraints," Journal of Guidance, Control, and Dynamics, vol. 37, no. 4, pp. 1114-1130, 2014.

[18] S. Ding, Z. Zhang, and X. Chen, "Guidance law design based on non-smooth control," Transactions of the Institute of Measurement and Control, vol. 35, no. 8, pp. 1116-1128, 2013.

[19] Z. Zhu, D. Xu, J. Liu, and Y. Xia, "Missile guidance law based on extended state observer," IEEE Transactions on Industrial Electronics, vol. 60, no. 12, pp. 5882-5891, 2013.

[20] J. Liu, S. Laghrouche, and M. Wack, "Adaptive higher order sliding mode observer based fault reconstruction for a class of nonlinear uncertain systems," in Proceedings of the 52nd IEEE Conference on Decision and Control, pp. 5668-5673, 2013.

[21] H. Alwi and C. Edwards, "Oscillatory failure case detection for aircraft using an adaptive sliding mode differentiator scheme," in Proceedings of the American Control Conference (ACC '11), pp. 1384-1389, July 2011.

[22] Y. Shtessel, M. Taleb, and F. Plestan, "A novel adaptive-gain supertwisting sliding mode controller: methodology and application," Automatica. A Journal of IFAC, the International Federation of Automatic Control, vol. 48, no. 5, pp. 759-769, 2012.

[23] Y. Zheng, J. Liu, X. Liu, D. Fang, and L. Wu, "Adaptive secondorder sliding mode control design for a class of nonlinear systems with unknown input," Mathematical Problems in Engineering, vol. 2015, Article ID 319495, 7 pages, 2015.

[24] Y. B. Shtessel, J. A. Moreno, F. Plestan, L. M. Fridman, and A. S. Poznyak, "Super-twisting adaptive sliding mode control: a Lyapunov design," in Proceedings of the 49th IEEE Conference on Decision and Control (CDC '10), pp. 5109-5113, Atlanta, Ga, USA, December 2010.

[25] A. Pisano and E. Usai, "Globally convergent real-time differentiation via second order sliding modes," International Journal of Systems Science, vol. 38, no. 10, pp. 833-844, 2007. 


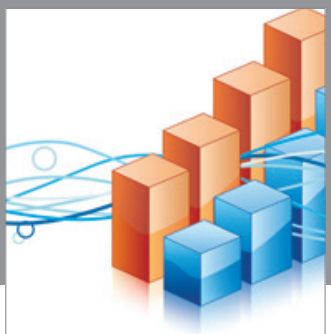

Advances in

Operations Research

vatem alat4

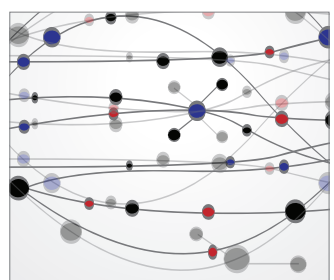

\section{The Scientific} World Journal
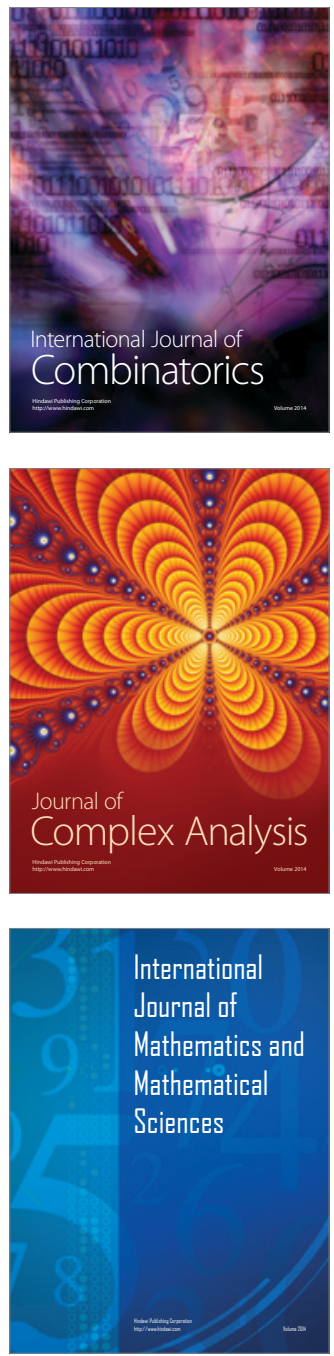
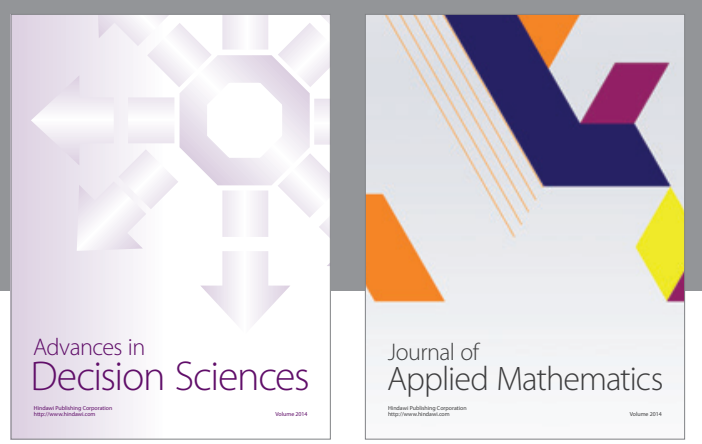

Algebra

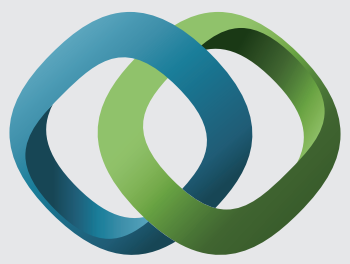

\section{Hindawi}

Submit your manuscripts at

https://www.hindawi.com
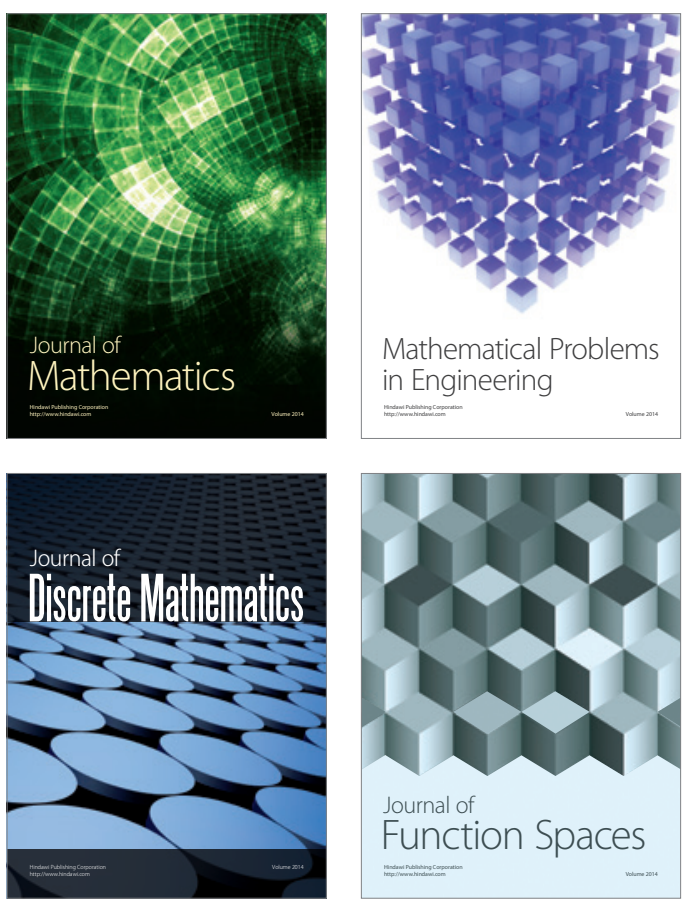

Mathematical Problems in Engineering
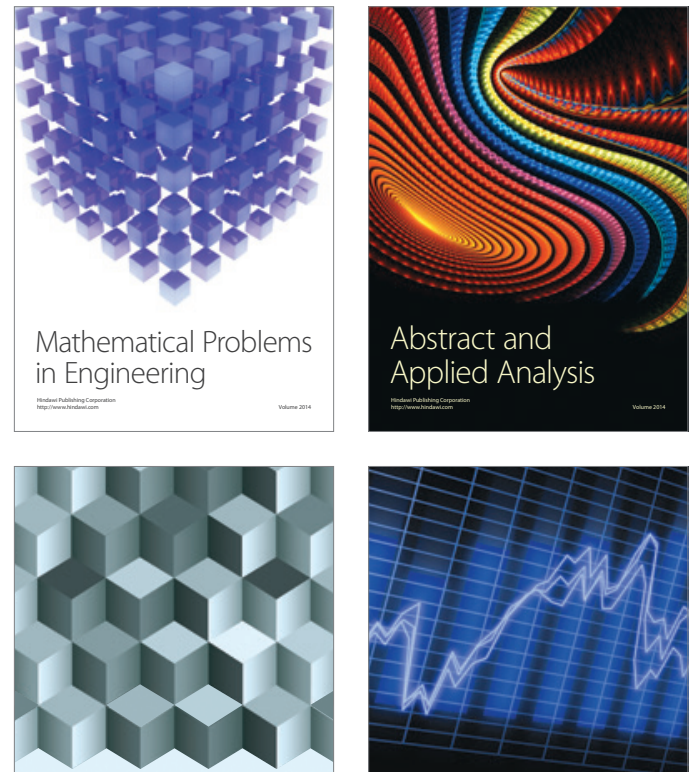

Journal of

Function Spaces

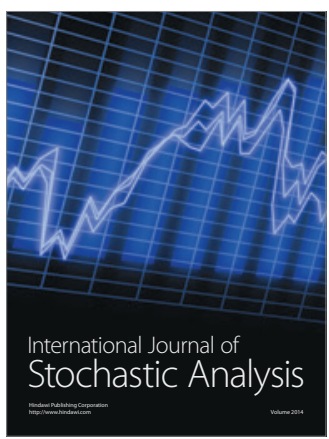

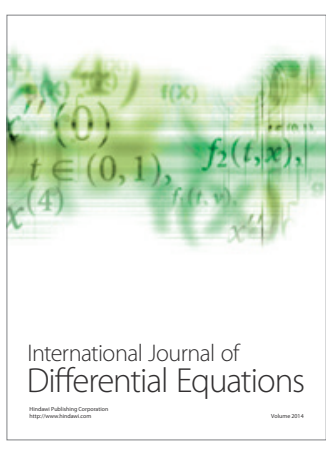
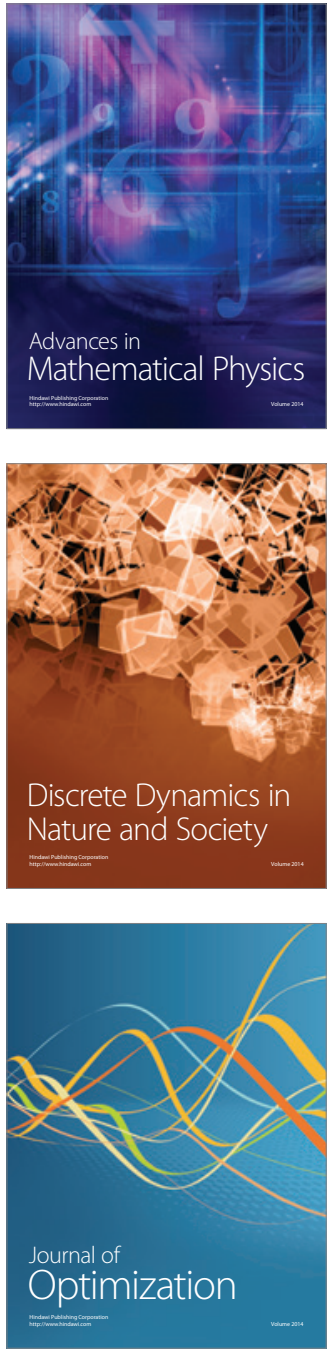